\begin{tabular}{|l|l|l|l|}
\hline Eiszeitalter u. Gegenwart & 35 & $\begin{array}{c}15-22 \\
4 \mathrm{fig} .\end{array}$ & Hannover 1985 \\
\hline
\end{tabular}

\title{
Variations du niveau marin a l'Holocène en Bretagne (France)
}

\author{
MARIe-Thérēse Morzadec-Kerfourn *)
}

\begin{abstract}
Borehole section, coastal environment, transgression, sea level change,
Holocene, pollen analysis, correlation.

Bretagne, Cotes du Nord (Dol region), English Channel, Gulf of St. Malo
\end{abstract}

$\mathrm{R}$ é s u $\mathrm{m}$ é : Les phases transgressives majeures qui se manifestent en Bretagne entre 5000 et 4400 B.P., 3300 et 3000 B. P. et autour de 1400 B. P. peuvent être comparées avec les phases Calais III, Dunkerque 0 et Dunkerque II définies aux Pays-Bas. Des incursions marines de moindre importance peuvent être observées en Bretagne, localement, en particulier dans les zones basses de la Baie du Mont-Saint-Michel.

[Holozäne Meeresspiegel-Schwankungen in der Bretagne (Frankreich]

$\mathrm{Kurz}$ a s s u $\mathrm{ng}$ : Die bedeutendsten transgressiven Phasen, die für die Bretagne zwischen 5000 und $4000 \mathrm{v}$. h., 3300 und $3000 \mathrm{v}$. h. und um $1400 \mathrm{v}$. h. angezeigt sind, lassen sich mit dem Calais III, Dunkerque 0 und Dunkerque II der niederländischen Holozän-Chronologie vergleichen. Weitere unbedeutende transgressive Phasen kommen in der Bretagne insbesondere in tief liegenden Regionen wie der Bucht von Mont-Saint-Michel vor.

\section{[Holocene Sea-Level Changes in Brittany (France)]}

A b s t a c t: The main transgressive phases known in Brittany: $5000-4400 \mathrm{~B}$. P., $3300-3000$ B. P., and around 1400 B. P. can be compared with the Calais III, Dunkerque 0 and Dunkerque II of the chronology of Holocene in the Netherlands. Other little transgressive phases are observed in Brittany, specially in low-land regions as the Mont-Saint-Michel bay.

La Bretagne présente une façade maritime importante, à morphologie diversifiée, dominée cependant par des falaises rocheuses. Les zones basses sont rares à l'exception de celles de l'estuaire de la Loire et de la Baie du Mont-Saint-Michel où d'ailleurs la falaise fossile, empâtée par des formations périglaciaires, demeure très proche en arrière des marais maritimes.

Le long du littoral breton l'amplitude de la marée subit de grandes variations; elle croît en Manche d'Ouest (6,50 m à Ouessant), en Est (13 m au Mont-Saint-Michel) et décroît en Atlantique d'Ouessant à l'estuaire de la Loire $(5 \mathrm{~m})$. Pour déterminer

*) Adresse de l'auteur: Dr. M.-T. Morzadec-Kerfourn, Institut de Géologie, Université de Rennes, F-35042 Rennes Cedex/Frankreich. 
la position des niveaux marins passés le point important est le passage du domaine maritime au domaine continental; il correspond au niveau des plus hautes mers de vives eaux. Il est aisément révélé par la faune, le plancton ou la végétation (MORZADECKerFourn 1979). Pour la Bretagne toutes les altitudes ont été déterminées dans les séquences flandriennes par rapport à ce changement de milieu, en utilisant en particulier la présence de kystes de Dinoflagellés qui témoigne du maintien d'une certaine salinité de l'eau. Etant donné l'importance des variations d'amplitude des marées en Bretagne, ce niveau repère est variable suivant la position géographique et la situation morphologique; d'où la nécessité de bien séparer les zones d'étude. Les résultats obtenus reflètent l'irrégularité de la remontée marine flandrienne, avec des phases transgressives plus accélérées (MORZADEC-KERFOURN 1969, 1974).

\section{Le marais maritime de Dol-de-Bretagne (Ille-et-Vilaine)}

La séquence flandrienne la plus complète provient du marais de Dol-de-Bretagne où la sédimentation s'est effectuée, tantôt dans le domaine intertidal, tantôt dans le domaine supratidal. Les niveaux tourbeux supratidaux sont séparés par des silts calcaires (tangue) déposés sur la slikke. Grâce à des datations radiocarbone (DÉlibrias \& MORZADEC-KERFourn 1975), il est possible d'envisager, à titre d'hypothèse, des corrélations avec les phases transgressives définies dans les séries flandriennes des Pays-Bas.

Le ralentissement de la transgression flandrienne se manifeste dans le marais de Dol par le passage d'une sédimentation sableuse à une sédimentation plus fine silteuse; l'influence marine cesse dans la région de Châteauneuf vers $5500 \pm 150$ (Gif 1837). Les marais se développent autour du Mont-Dol entre $5450 \pm 150$ (Gif 948) et $5000 \pm 150$ (Gif 944). Lors de la phase transgressive qui suit (Calais III?) la mer atteint le point le plus avancé à l'intérieur des terres; elle pénètre dans le Biez-Jean, jusqu'à Vildé-Bidon entre $4800 \pm 140$ (Gif 953) et $4400 \pm 140$ (Gif 1835) (fig. 1). Les marais regagnent ensuite vers le Nord entre $3900 \pm 140$ (Gif 950) et $3450 \pm 100$ (Gif 947). Une transgression importante (Dunkerque 0?) entre 3400 et 3000 B.P. recouvre une grande partie des marais. Les silts qui se déposent, constituent la surface actuelle autour du Mont-Dol; ils sont colonisés dès $3000 \pm 100$ (Gif 2185) par une végétation halophile et de sables fixés. Aucune phase transgressive plus récente n'apparaît dans ce secteur.

Cependant à l'Ouest, dans l'estuaire du Biez-Jean, comme à l'Est, dans l'estuaire du Couesnon, des oscillations marines plus récentes se sont faites sentir. Une transgression post-romaine avait été mise en évidence dans l'estuaire du Couesnon, à Pontaubault par LeBesConTE (1898); elle a été confirmée par des travaux récents et rapportée au Dunkerquien II (Clet-Pellerin, Lautridou \& Délibrias 1981).

Vers l'Ouest, près de l'estuaire du Biez-Jean, à la Fresnais dans la partie supérieure du sondage, un niveau riche en matière organique est interstratifié entre des silts calcaires à microfaune littorale et à pollens de Chénopodiacées uniquement (cf. base du diagramme fig. 2). La tourbe supérieure vers $1,30 \mathrm{~m}$ a livré une céramique gauloise datant du premier siècle de notre ère. Le milieu, une Aulnaie à Bouleau, est nettement continental; il est caractérisé par une faible représentation des Chénopodiacées, l'absence de kystes de Dinoflagellés, le développement des Phragmites et un début $d$ 'installation de Sphagnum. La mise en place du silt calcaire supérieur ( $t$, fig. 3 ) est 
MONT - DOL

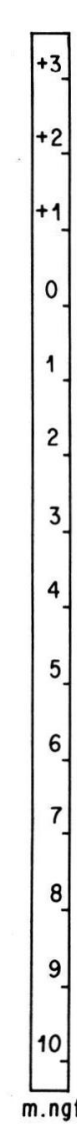

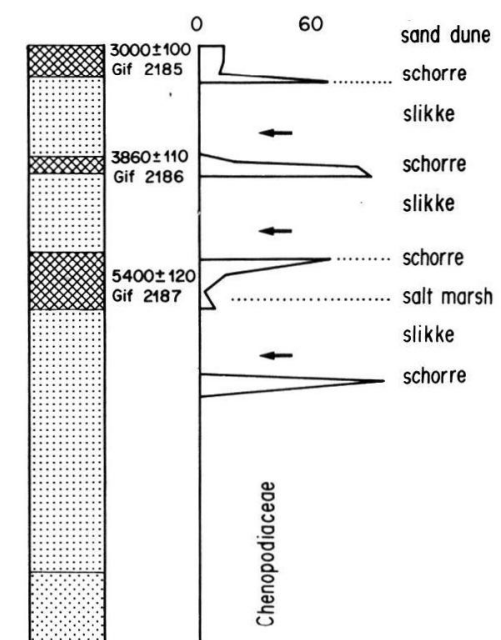
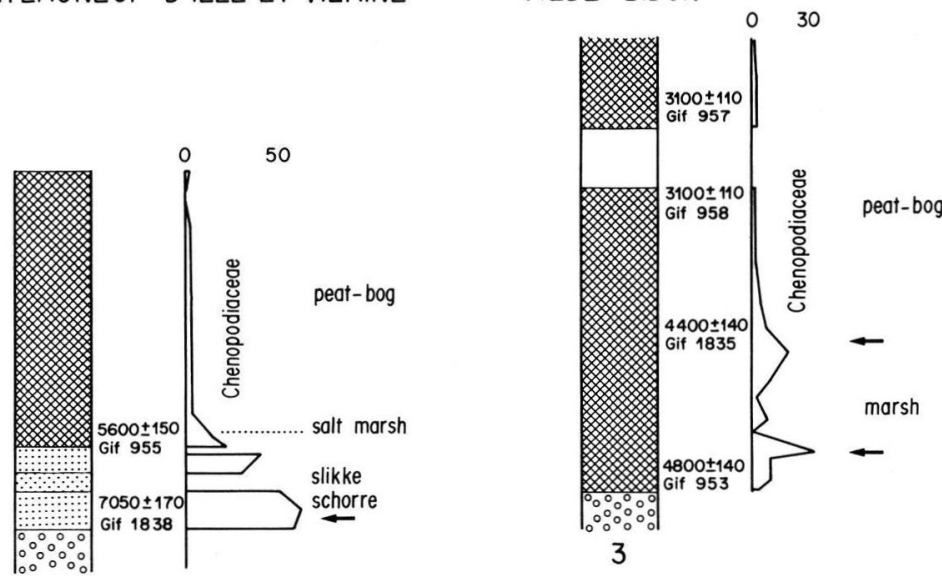

3

2

Chenopodiacea $=\%$ of total pollen

intertidal zone
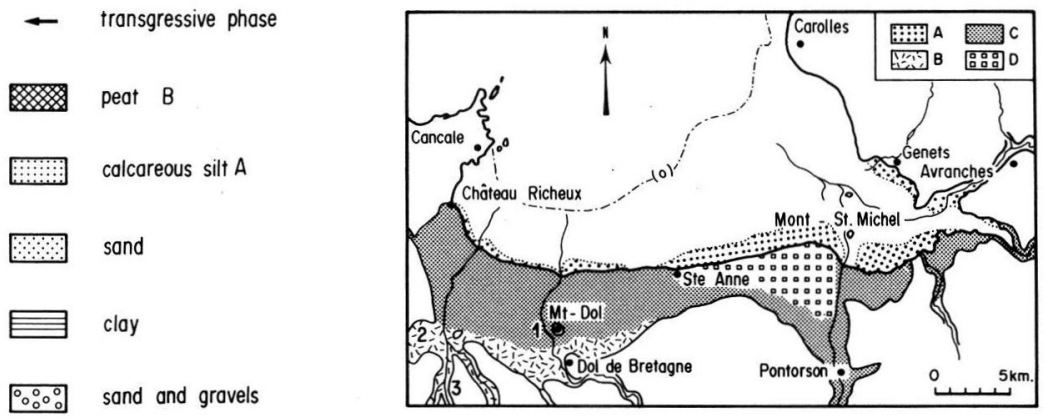

है.

Fig. 1: La marais de Dol-de-Bretagne (Ille-et-Vilaine). Phases transgressives dans trois sondages.

Fig. 1: Dol-de-Bretagne Marshes (Ille-et-Vilaine). Transgressives phases in three core profiles. 


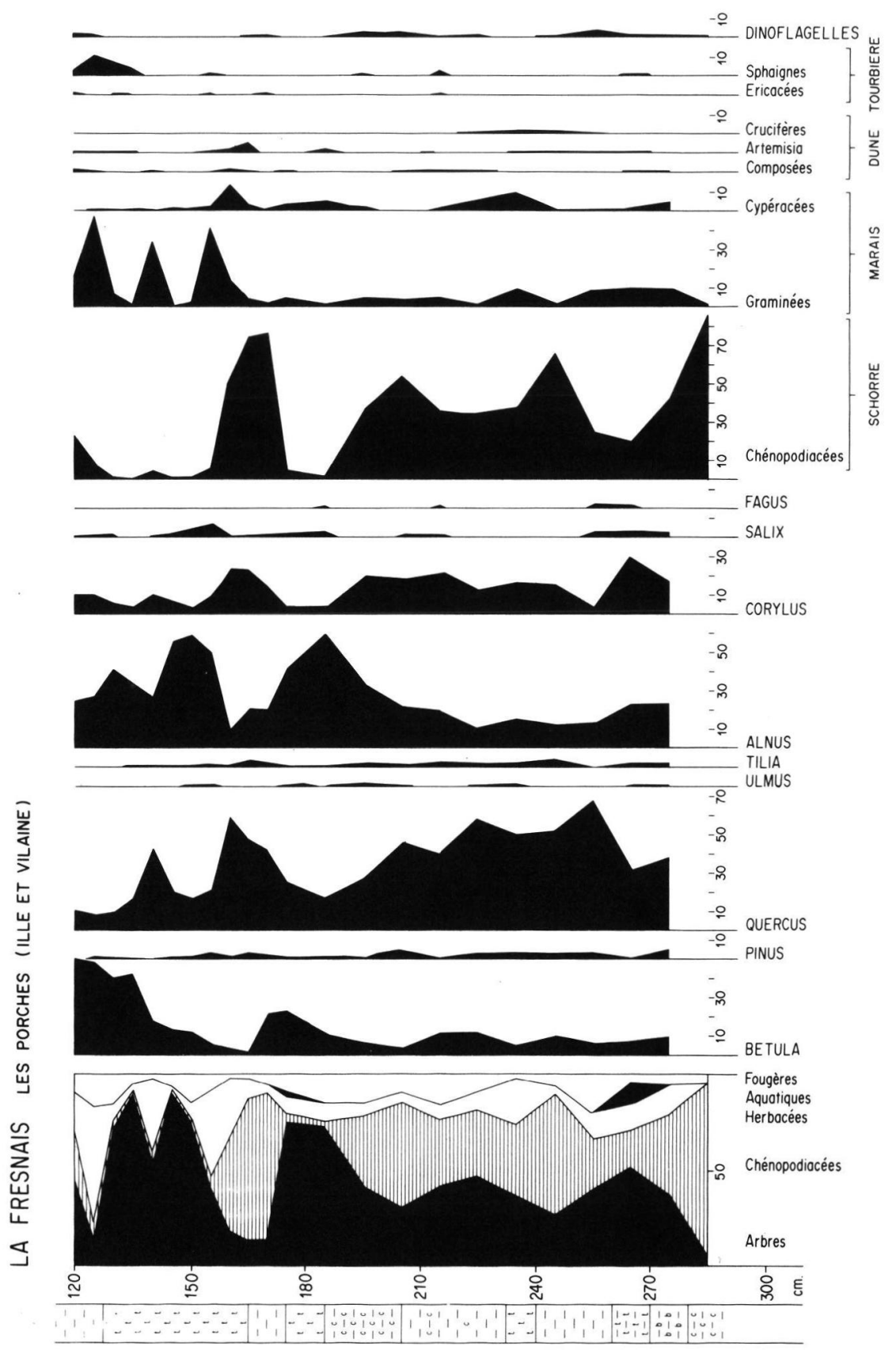

Fig. 2: Diagramme pollinique de la partie supérieure du Sondage de la Fresnais-Ouest du Mont-Dol.

Fig. 2: Pollen-analyses of the upper part of the Fresnais core - West of the Mont-Dol. 


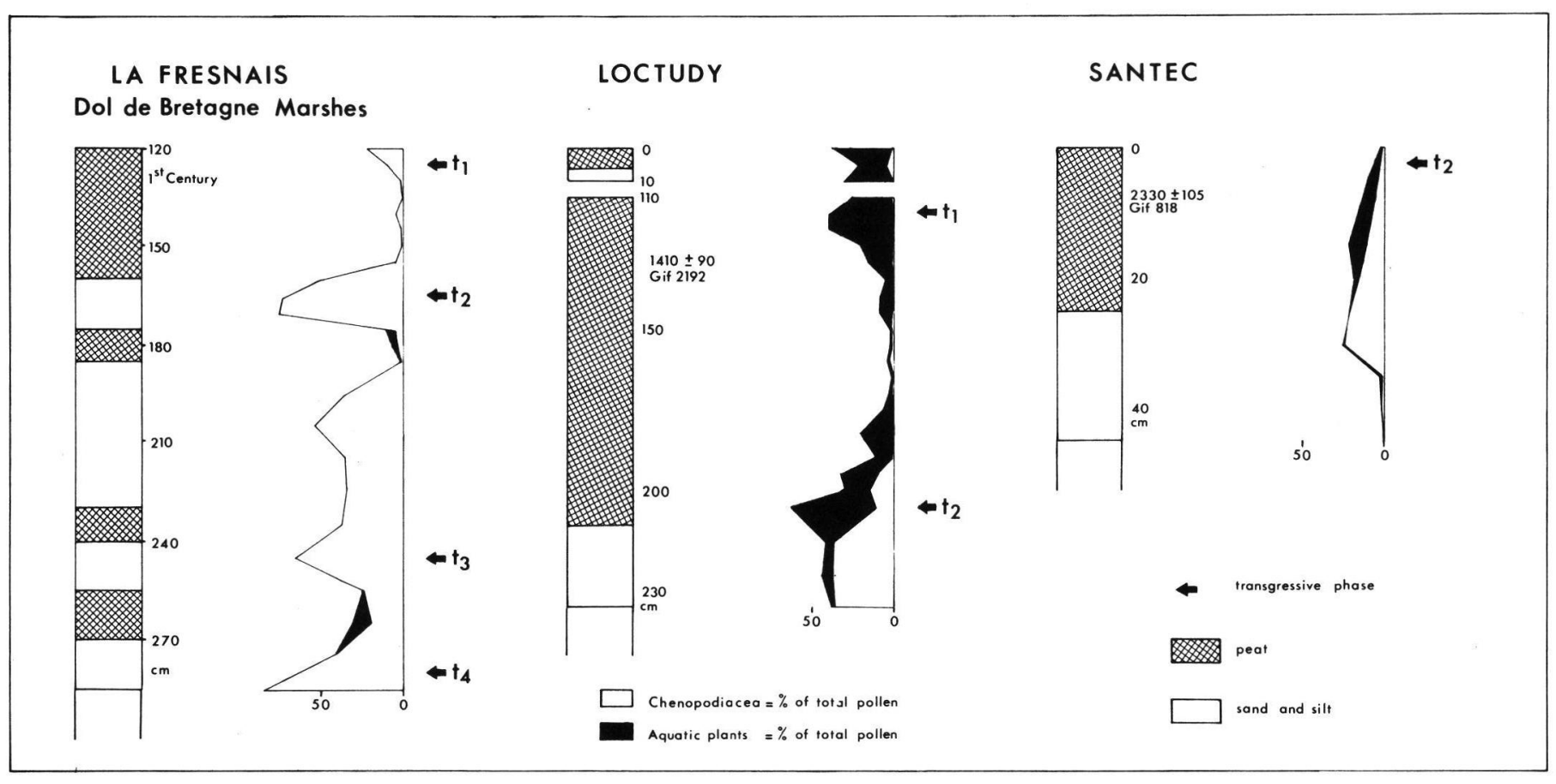

Fig. 3: Phases transgressives récentes dans trois sites de Bretagne:

La Fresnais, marais de Dol-de-Bretagne; Loctudy, Sud Finistère; Santec, Nord Finistère. Représentation des pollens de Chénopodiacées et de plantes aquatiques.

Fig. 3: Recent transgressive phases in three locality of Brittany: La Fresnais, Dol-de-Bretagne marshes, Loctudy, south of Finistère coast, Santec, North of Finistère coast. Distribution of the pollen grains of Chaenopodiacae and aquatic plants. 

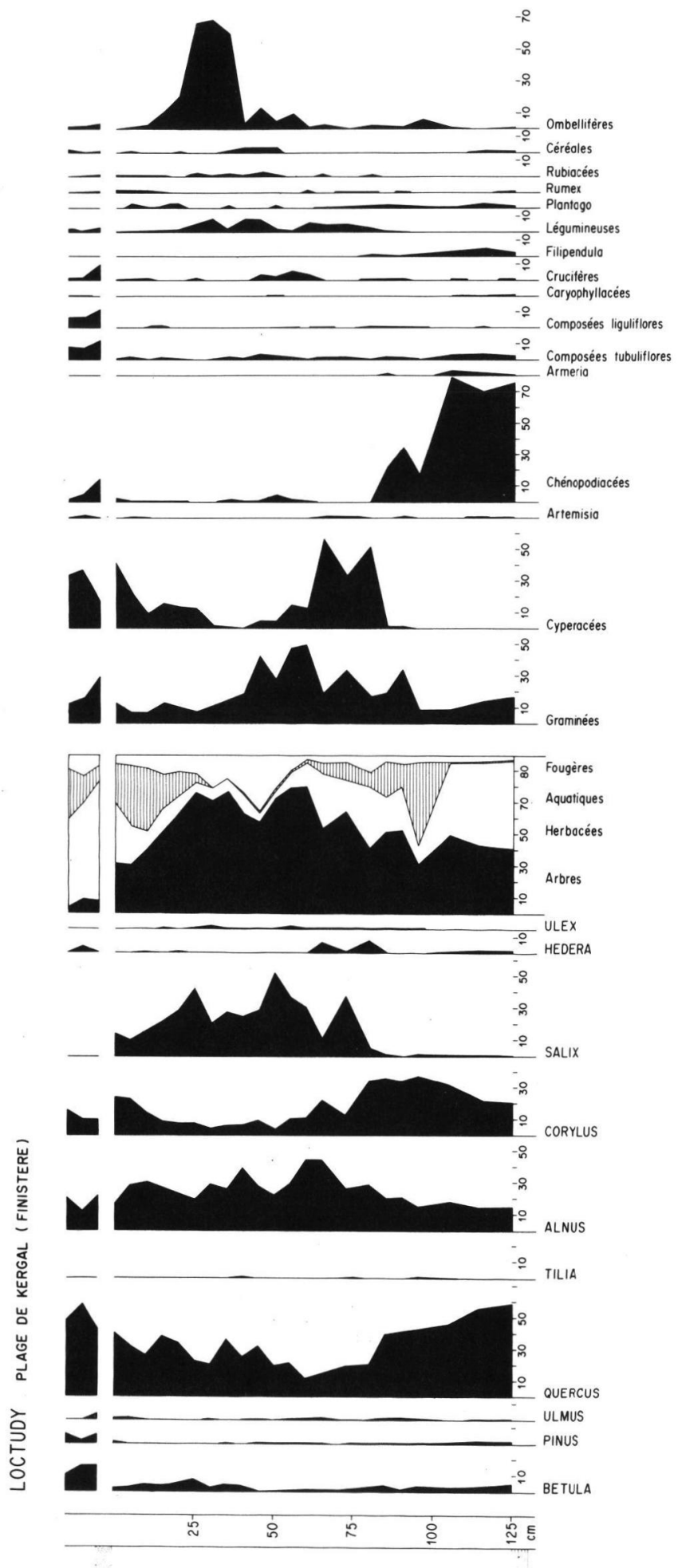
donc, comme dans l'estuaire du Couesnon, contemporain du Dunkerquien II. Le silt inférieur ( $t 4$, fig. 3) est à rapporter au niveau supérieur des sondages réalisés autour du Mont-Dol, déposé entre 3450 B.P. et 3000 B.P. Entre ces deux phases transgressives majeures, les incursions marines ( $\mathrm{t} 3$ et $\mathrm{t} 2$, fig. 3 ) pourraient être des manifestations comparables aux différentes phases du Dunkerquien 1 aux Pays-Bas. La transgression t2, en particulier, a submergé un marais à Aulne et Bouleau (entre 1,70 et 1,60 m fig. 2), déjà bien établi, ce qui nécessite une variation d'amplitude du niveau marin relativement importante. Cependant les manifestations de cette transgression sont minimes dans l'ensemble de la Baie du Mont-Saint-Michel; elles n'affectent que les estuaires.

\section{Le littoral rocheux}

Sur les platiers rocheux, en avant des falaises, les dépôts tourbeux débutent peu après 6000 B. P. Ils s'échelonnent sur les estrans entre le niveau des basses mers et celui des hautes mers actuelles. Ce sont les témoins de petits marais littoraux formés derrière des cordons de barrage. Les dépôts tourbeux peuvent être regroupés en trois ensembles suivant leur position (MOZARDEC-KeRFOURN 1969, 1974).

Pour le premier, situé entre le niveau des basses mers et le niveau moyen, les dates s'échelonnent entre 5700 et 4900 B. P.; des dépôts situés vers le niveau moyen fournissent des dates comprises entre 4250 et 3400 B.P., ils sont contemporains des allées couvertes submergées de la fin du Néolithique; les dépôts situés au-dessus du niveau moyen sont souvent associés à des formations dunaires; ils ont été datés de 2330 à 1400 B.P.

Les dépôts tourbeux les plus récents montrent l'évolution des zones humides littorales en relation avec la transgression flandrienne. La tourbe de Loctudy (Finistère) affleure sur l'estran vers le niveau moyen des marées; un niveau supérieur épais de $20 \mathrm{~cm}$ apparaît au niveau des plus hautes mers de mortes eaux. Après la formation du cordon sableux de barrage, le mauvais écoulement des eaux douces se traduit par une régression des pollens de Chénopodiacées, une augmentation des aquatiques (fig. 4). Puis la progression marine se ralentissant, les Cypéracées et les Phragmites colonisent le marais suivies par une végétation de sables fixés à Ombellifêres. Une nouvelle progression marine modifie l'équilibre du marais; les aquatiques se développent puis ensuite les Cypéracées. Cette modification de l'équilibre biologique du marais peut être assimilé à une phase transgressive; elle a été datée de $1410 \pm 90$ (Gif 2192); elle est donc contemporaine de la phase t 1 mise en évidence dans le sondage de la Fresnais (Fig. 3). Une évolution comparable se retrouve dans la tourbe de Santec (Finistère) (MorZADec-Kerfourn 1974). Elle a été datée de $2330 \pm 105$ (Gif 818); elle serait donc contemporaine de la phase inférieure de la tourbe de Loctudy. Un silt gris, représentant un ancien schorre affleure sur la plage de Plounéour-Trez (Finistère) à $2 \mathrm{~m}$ au-dessus du niveau moyen des marées (MorZADEC-Kerfourn 1974). Il a été daté de $1390 \pm 100$ (Gif 1300) et semble indiquer que la transgression assimilée au Dunkerquien II n'a pas dépassé le niveau actuel.

Fig. 4: Diagramme pollinique de la tourbe de la plage de Loctudy (Finistère).

Fig. 4: Pollen-analysis of the peat exposed on the beach of Loctudy (Finistère). 


\section{Conclusion}

Cet essai de comparaison entre les résultats obtenus aux Pays-Bas et en Bretagne montre qu'il y a, en envisageant seulement les grandes phases de transgression, une certaine convergence (HAGEMAN 1969, ROELEVELD 1974, MORZADEC-KeRFOURN 1974), alors que l'examen des courbes de variations du niveau marin proposées, basées sur des altitudes difficiles à préciser et dont la signification varie suivant les conditions morphologiques, ne permet pas une telle conclusion. Ceci tendrait à prouver qu'en dehors des effets locaux liés à la configuration des littoraux il y a des phénomènes plus généraux, certainement des accélérations rapides de la transgression, qui font que marais et estran se succédent périodiquement en un même point.

\section{Remerciements}

La découverte de la poterie de la Fresnais a été signalée par J. Meury qui a également réalisé le sondage de la Pigassière. La poterie a été étudiée par L. LANGOUËT (travail à paraître). Les préparations palynologiques des sédiments de la Fresnais et de Loctudy ont été faites dans le cadre d'une action concertée de la D.G.R.S.T.: Archéologie du Paysage Armoricain; responsable P. R. GıoT. Toutes les datations radiocarbone ont été effectuées à Gif-sur-Yvette par G. DéLibRIAS.

\section{Références bibliographiques}

Clet-Pellerin, M., Lautridou, J. P. \& Délibrias, G. (1981): Les Formations holocènes et Pleistocènes de la partie orientale de la baie du Mont-Saint-Michel. - Bull. Soc. Linn. Normandie, 109: 3-20; Caen.

Délibrias, G. \& Morzadec-Kerfourn, M. T. (1975): Evolution du marais de Dol-de-Bretagne au Flandrien (Ille-et-Vilaine, France). - Bull. A. F.E.Q., 2: 59-70; Paris.

Hageman, B. P. (1969): Development of the Western part of the Netherlands during the Holocene. - Geol. en Mijnbown, 48: 373-388; Leiden.

Jelgersma, S. (1961): Holocene Sea-level changes in the Netherlands. - Mededel. Geol. Stichting C, VI, 7: 100 pp.; Maastricht.

Lebesconte, P. (1898): Périodes géologiques gallo-romaine et franque. Leurs relations avec le Quaternaire, le Pliocène et l'Epoque moderne. — Bull. Soc. Sci. medic. Ouest, 7: 354-408; Nantes.

Morzadec-Kerfourn, M. T. (1974): Variations de la ligne de rivage armoricaine au Quaternaire. Analyses polliniques de dépôts organiques littoraux. Mémoires Soc. géol. minér. Bretagne, 17: 280 p. (Thèse); Rennes.

- (1979): Indicateurs écologiques du domaine littoral: Végétation et Plancton organique. - Océanis, 5, H. S.: 207-213; Paris.

Roeleveld, W. (1974): The Groningen Coastal Area: A study in Holocene geology and low-land physical geography. - Berichten von de Rijksdienst voor bet Oud heid kundig Bodemonderzoek, 24: 7-132: supplement; Amsterdam. 